\title{
Antibacterial Activity of Essential Oils in Aromatherapy Protocols
}

\author{
Yago Queiroz dos Santos ${ }^{1,4 *}$, Gabriella Silva Campos Carelli ${ }^{2,4}$, Bruno Oliveira de $\operatorname{Veras}^{3}$ and \\ Elizeu Antunes dos Santos ${ }^{2}$ \\ ${ }^{1}$ Federal Institute of Education, Science and Technology of Ceará, Brazil \\ ${ }^{2}$ Department of Biochemistry, Federal University of Rio Grande do Norte, Brazil \\ ${ }^{3}$ Department of Biochemistry, Federal University of Pernambuco, Recife, Pernambuco, Brazil \\ ${ }^{4}$ Institute of Tropical Medicine, Federal University of Rio Grande do Norte, Brazil
}

*Corresponding author: Yago Queiroz dos Santos, Federal Institute of Education, Science and Technology of Ceará, Boa Viagem, Ceará, Brazil.

To Cite This Article: Yago Queiroz dos Santos. Antibacterial Activity of Essential Oils in Aromatherapy Protocols. Am J Biomed Sci \& Res. 2019 5(2). AJBSR.MS.ID.000885. DOI: 10.34297/AJBSR.2019.05.000885.

Received: 眥 September 5, 2019; Published: 眥 September 12, 2019

\begin{abstract}
Aromatherapy is the therapy that employs volatile aromatic elements of the plants, that is, their essential oils. The practice that helps patients to sleep and rest as well as helps on alertness, creativity, among others. The therapeutic massage integrates a set of practices with maneuvers whose goal is to promote health and balance with the body, promoting psychological effects on the skin, visceral pain. This pharmacological components of essential oils are volatile constituents at temperature environment, most of which originate from secondary metabolism produced and stored in their own secretory structures formed in the leaves, flowers, branches, stems or roots of various species usually secreted by glandular trichomes, which have various forms, structures and functions distributed mainly on the surface of the leaves. The identification of essential oil constituents is important for the understanding and prediction of their physiological effects where main studied activities are antimicrobial activity, namely antibacterial, antifungal and antiviral, anxiolytic, antioxidant, anticarcinogenic and antinociceptive effects helpful to develop new clinical aromatherapy protocols.
\end{abstract}

Keywords: Essential Oil; Plant; Antibacterial

\section{Background}

Aromatherapy consists of a branch of Phyto therapy that is based on the premise of essential oils as therapeutic agents in promoting and maintaining the well-being of the Man, who goes through the treatment and prevention of diseases, as well as in the treatment of altered emotional states. This therapy is seen as a complementary medicine, used mostly in primary health care being compatible with classical therapy. Aromatherapy is a natural and non-invasive practice, designed to not only act on the symptom or disease, but also to maintain the natural balance of the organism, by the correct use of the essential oils. Such a definition gives the holistic view of this therapy, that is, it addresses the whole body including the physical and mental part of the individual [1].

Essential Oils (EO) are mixtures of volatile constituents at temperature environment, most of which originate from secondary metabolism. produced and stored in their own secretory structures formed in the leaves, flowers, branches/stems or roots of various species. In various plant families, namely Lamiaceae and Verbenaceae, usually secreted by glandular trichomes, which have various forms, structures and functions distributed mainly on the surface of the leaves [2]. As specialized cell structures, trichomes, such as organs of EO synthesis and protection, protect the plant from its own toxicity certain constituents, such as monoterpenes, which concentrations are phytotoxic. Lamiaceae trichomes are of two types morphologically distinct: naked and pilous, distinguished by their structure and form of secretion, a fact evidenced by microscopic observations optics and electronics [3] (Figure 1).

The use of these plant products has started from popular wisdom, since the beginning of time, for the complementary therapies that marked the resurgence of these products. Nowadays, essential oils exhibit a high response to a variety of situations where people's health and well-being committed. The risks associated with chemically synthesized drugs, adverse effects of various therapies, the existing treatments, and the imminent lack of curbing these diseases, have driven the search for new alternatives [4].

The biochemical defense mechanisms against pathogens are broad (Figure 1), highly dynamic and are mediated by responses 
that are either directly or indirectly [5], since the defensive compounds like Essential Oils can be produced in a constant and ubiquitous manner throughout the plant have many possibilities and an enormous potential to improve our clinical with new reservoir of molecules capable to give new treatments to well established human microbial diseases.

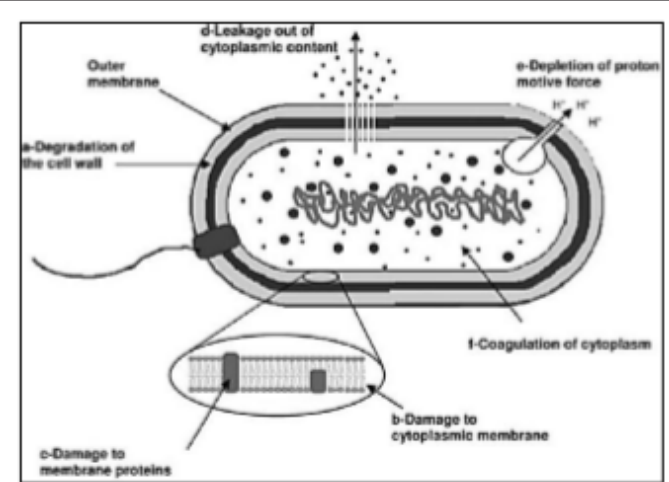

Figure 1: Antibacterial Mechanisms of Essential Oils.

\section{References}

1. Lis-Balchin M (2010) Handbook of essential oils-Science, Technology and Applications. Baser KHC, Buchbauer G (eds) EUA Taylor \& Francis Group pp. 549-584.

2. Burdock GA, Carabin IG (2008) Safety assessment of sandalwood oil (Santalum album L) Food Chem Toxicol 46(2): 421-432.
3. Hayouni EA, Chraief I, Abedrabba M, Bouix M, Leveau JY, et al. (2008) Tunisian Salvia officinalis L and Schinus molle L essential oils: their chemical compositions and their preservative effects against Salmonella inoculated in minced beef meat. Int J Food Microbiol 125(3): 242-251.

4. Sindhu RK, Upma, Ashok Kumar, Sahil Arora (2010) Santalum album Linn: a review on morphology, phytochemistry and pharmacological aspects. International Journal of Pharm Tech Research 2(1): 914-919.

5. Dang L, Damme, EJM Van (2015) Toxic proteins in plants. Phytochemistry 117: 51-64. 